\title{
Effects of Klebsiella pneumoniae on Toll-Like Receptor-Dependent Endoplasmic Reticulum Stress-Related Signaling Pathways and Gene Expression and Promotes HLA-B27 Misfolding
}

Wen-Jen Cheng ${ }^{1}$, Yu-Ling Wei ${ }^{2}$, James Cheng-Chung Wei ${ }^{2,3,4}$, Chia-Wei Lin ${ }^{5}$, Hui-Yin Lu $^{6}$, Yu-Pu Hsia ${ }^{6}$, Pei-Jane Tsai ${ }^{7}$, Ying-Chun Lai ${ }^{8}$, Yu-Liang Kuo ${ }^{9,10}$ and Ming-Shiou Jan ${ }^{4,5,11}$

1. Department of Emergency, Antai Tian-Sheng Memorial Hospital, Ping Tong 928, Taiwan, R.O.C.

2. Institute of Medicine, Chung Shan Medical University, Taichung 402, Taiwan, R.O.C.

3. Graduate Institute of Integrated Medicine, China Medical University, Taichung 404, Taiwan, R.O.C.

4. Division of Allergy, Immunology and Rheumatology, Chung Shan Medical University Hospital, Taichung 402, Taiwan, R.O.C.

5. Institute of Biochemistry, Microbiology and Immunology, Chung Shan Medical University, Taichung 402, Taiwan, R.O.C.

6. Department of Biomedical Sciences, Chung Shan Medical University, Taichung 402, Taiwan, R.O.C.

7. Department of Medical Laboratory Science and Biotechnology, National Cheng Kung University, Tainan 701, Taiwan, R.O.C.

8. School of Applied Foreign Languages, Chung Shan Medical University, Taichung 402, Taiwan, R.O.C.

9. Department of Medical Imaging, Chung Shan Medical University Hospital, Taichung 402, Taiwan, R.O.C.

10. School of Medical Imaging and Radiological Sciences, Chung Shan Medical University, Taichung 402, Taiwan, R.O.C.

11. Immunology Research Center, Chung Shan Medical University, Taichung 402, Taiwan, R.O.C.

\begin{abstract}
Klebsiella has been considered as initiator of AS (ankylosing spondylitis) for nearly four decades. This study aimed to demonstrate that Klebsiella triggers ERS (endoplasmic reticulum stress) and HLA-B27 heavy chain misfolding. CA46 cells or splenocytes obtained from wild-type, MyD88 ${ }^{-/}$or TLR $9^{-/-}$mice were stimulated with KP (Klebsiella pneumoniae) or its components including CPS (capsule polysaccharide), LPS (lipopolysaccharide), and KP gDNA (genomic deoxyribonucleic acid) respectively for $24 \mathrm{~h}$ and $48 \mathrm{~h}$. The activation of ERS-related signaling was detected by Western blotting or RT-PCR, and the level of misfolded HLA-B27 was determined by non-reducing protein gel electrophoresis and Western blotting. The protein expression of BiP/Grp78 and calreticulin, the alternative splicing of XBP-1 mRNA (messenger ribonucleic acid), and the activation of caspase-12 and $\mathrm{p} 38$ were increased in a dose-dependent manner in HLA-B27-expressing CA46 cells after treatment with decapsulated KP. We also demonstrate that the ERS-inducing effects occur via the TLR (Toll-like receptor)/MyD88-dependent signaling pathway. Significantly, HLA-B27 misfolding was also detected in decapsulated KP-treated B27-expressing cells. These results suggest that the non-antigen-specific induction of ERS and B27 misfolding through TLR/MyD88 signaling might promote KP antigen-initiated autoreactive responses via the presentation of misfolded B27, and that small-molecules targeting TLRs might have potential as novel therapeutic agents for AS.
\end{abstract}

Key words: Klebsiella pneumoniae, endoplasmic reticulum stress, ankylosing spondylitis, Toll-like receptor 9, MyD88, HLA-B27 misfolding.

\section{Introduction}

Autoimmune diseases are characterized by attacks

Corresponding author: Ming-Shiou Jan, Ph.D., associate professor, research fields: pathogenesis of autoimmune and pancreatic cancer. on tissues or cells by the self-immune system via auto-recognition. The contemporary theories suggest that at least three key elements (genetic background, environmental factors and immune dysregulation) lead to the pathogenesis of autoimmune diseases. Among 
the relevant environmental factors, infection is most extensively studied. Pathogen infection might cause disruption of the physiological barriers that prevent self-antigen recognition, resulting in the release of self-antigens from damaged cells or the induction of self-reactive immunity via a pathogen-derived peptide that mimics a self-peptide [1]. The most direct evidence indicating a correlation between microbes and autoimmunity was based on studies comparing autoimmune-prone animals housed in a SPF (specific antigen-free) environment or germ-free conditions. The autoimmune symptoms observed in SPF rodents are not observed in germ-free rodents [2, 3]. PRRs (pattern recognition receptors) expressed on/in immune cells stimulate cell activation via their ligands, PAMPs (pathogen-associated molecular patterns) or DAMPs (damage-associated molecular patterns). Most TLRs (toll-like receptors, a PRR family) induce inflammatory signaling pathways involving NF- $\mathrm{B}$ and AP-1 via MyD88. In addition to the antigen mimicry theory of the induction of autoreactive immunity, PAMPs function as adjuvants in the inflammatory response through the activation of innate immunity, which causes disruption of barriers and induction of cell damage. To date, many experimental autoimmune animal models involving induction by PAMPs have been developed for the study of autoimmune diseases [2, 4, 5].

AS (ankylosing spondylitis) is characterized by chronic inflammation and bone formation in vertebral bodies and adjacent enthesis throughout the spine $[6,7]$. Although AS is strongly associated with the HLA-B27 gene, only 5 10\% of HLA-B27-positive individuals develop AS, indicating that other factors, including environmental factors, contribute to AS pathogenesis. Several non-HLA-B27 genes, such as ERAP1 and STAT2/3, have been implicated in the pathogenesis of AS through genome-wide screening studies [8-12]. In addition, a role of immune responses against bacteria, particularly KP (Klebsiella pneumoniae), in AS has been discussed for four decades [13]. At least four hypotheses have been proposed for the etiology of AS: the molecular mimicry theory, the arthritogenic peptide theory, the ERS (endoplasmic reticulum stress) and UPR (unfolded protein response) hypothesis, and immune dysregulation through non-HLA gene polymorphism [12, 14-16].

$\mathrm{KP}$ is a Gram-negative enteric member of the normal flora that can cause primary liver abscess and pneumonia [17]. Although many virulence factors contribute to the pathogenesis of KP infection, the sticky CPS (capsular polysaccharide) plays the greatest role in liver abscess because of its anti-engulfment effect [18]. Since the 1970s, several studies have detected immunological cross-reactivity between antigens of Klebsiella spp. and HLA-B27 molecules. This discovery suggests that immune responses against Klebsiella contribute to the pathogenesis of AS. The molecular mimicry theory posits amino acid sequence similarities between several KP proteins, including pullulanases $\mathrm{D}$ and $\mathrm{A}$, and self-peptides in the HLA-B27 heavy chain or in types I, III, and IV collagen [14].

It had also been proposed that urogenital pathogens, not only focus on KP but also contribute to non-antigen-specific immune responses caused by PAMPs and/or DAMPs (damage-associated molecular patterns), and this hypothesis further explains why the inflammation sites observed in AS are primarily located in the spine and sacroiliac joints [19].

In the present study, we elucidated the non-antigenic effect of KP on ERS and HLA-B27 misfolding.

\section{Materials and Methods}

\subsection{Bacterial Culture and Capsule Polysaccharide Separation}

The KP CG43 strain was obtained from Dr. Y. C. Lai, Department of Microbiology and Immunology, Chung Shan Medical University, Taichung, Taiwan. 
KP CG43 was cultured in standard LB (Lysogeny broth) medium. To obtain the CPS and decapsulated bacteria, a $10-\mathrm{mL}$ overnight culture of bacteria was combined with $2 \mathrm{~mL}$ of 3-14 Zwittergent (1\% N-tetradecyl-N,N-dimethyl-3-ammonio-1-propanesulfonate (Cat. No. T-7763, Sigma, MO, USA) in citric acid buffer). After incubation at $50{ }^{\circ} \mathrm{C}$ for $20 \mathrm{~min}$, the samples were centrifuged at 10,000 rpm (revolutions per minute) for $20 \mathrm{~min}$ at room temperature. The CPS in the supernatant fraction was precipitated by adding absolute ethanol and subsequent freezing at $-20{ }^{\circ} \mathrm{C}$ for $30 \mathrm{~min}$. After centrifugation at $13,000 \mathrm{rpm}$ for $20 \mathrm{~min}$ at room temperature, the CPS pellets were washed twice with $80 \%$ ethanol and then air-dried. The decapsulated KP bacterial pellets were subsequently washed twice with $1 \times$ sterile PBS and air-dried.

The bacterial concentration was calculated by serially diluting the original bacterial culture and smearing $0.1 \mathrm{~mL}$ of the diluted bacterial suspension on three LB agar plates. After overnight incubation at $37{ }^{\circ} \mathrm{C}$ overnight, the bacterial colonies were counted, and the original bacterial concentration $(\mathrm{CFU} / \mathrm{mL})$ was calculated.

$\begin{array}{llrrr}\text { 2.2. } \quad \text { Extraction of } & K P & \text { Genomic } & \text { DNA } \\ \text { (Deoxyribonucleic } & \text { Acid) } & \text { and } & \text { LPS } \\ \text { (Lipopolysaccharide) } & & & \end{array}$

DNA (deoxyribonucleic acid) and LPS (lipopolysaccharide) were extracted using the Wizard ${ }^{\circledR}$ Genomic DNA purification kit (Cat. No. A1120, Promega, WI, USA) and an LPS extraction kit (Cat. No. 17141, iNtRON Biotechnology, Kyungki-Do, Korea) respectively, according to the manufacturer's instructions.

\subsection{Cells}

HLA-B27-bearing B cell lymphoma CA46 cells (ATCC No. CRL-1648) and U937 cells (ATCC No. CRL-1593.2 ${ }^{\mathrm{TM}}$ ) were purchased from BCRC (Bioresource Collection and Research Center, Taiwan) and maintained in RPMI 1640 with $10 \%$ heat-inactivated FBS (fetal calf serum) and $50 \mu \mathrm{g} / \mathrm{mL}$ gentamicin at $37{ }^{\circ} \mathrm{C}$ in an incubator with a humidified atmosphere with $5 \% \mathrm{CO}_{2}$. For stimulation with $\mathrm{KP}$ or its components, $1 \times 10^{6}$ cells $/ \mathrm{mL}$ were co-cultured with the indicated concentration of the bacterium or its components, including CPS, LPS, and gDNA (genomic deoxyribonucleic acid), for $24 \mathrm{~h}$ or $48 \mathrm{~h}$.

\subsection{Animals}

Specific-pathogen-free C57BL/6 mice were purchased from the National Laboratory Animal Center of Taiwan. MyD88 ${ }^{-/ /}$and TLR9 ${ }^{-/-}$mice were obtained from Dr. P. J. Tsai, Department of Medical Laboratory Science and Biotechnology, National Cheng Kung University, Tainan, Taiwan. All animals were housed in individually ventilated cages, fed a sterile diet and given sterile drinking water. The Institutional Animal Care and Use Committee of Chung Shan Medical University, Taichung, Taiwan, approved all experiments involving animals (IACUC Approval No. 789).

\subsection{Splenocyte Preparation and Cultivation}

Under sterile surgical conditions, the mouse spleens were removed and homogenized to prepare single-cell suspensions. After a hypotonic shock procedure to remove red blood cells, the splenocytes were resuspended in complete culture medium containing RPMI-1640, 10\% FBS, 2 mM L-glutamine, $1 \mathrm{mM}$ sodium pyruvate, $50 \mu \mathrm{M}$ 2-mercaptoethanol and $100 \mathrm{U} / \mathrm{mL}$ penicillin $/ 100 \mu \mathrm{g} / \mathrm{mL}$ streptomycin and co-cultured with the indicated doses of decapsulated bacteria, gDNA or LPS in a humidified atmosphere with $5 \% \mathrm{CO}_{2}$ at $37^{\circ} \mathrm{C}$ for $24 \mathrm{~h}$.

\subsection{Protein Gel Electrophoresis and Western Blot} Analysis

For the detection of protein expression, total cell lysates were prepared, and Western blotting was performed. Briefly, cell extracts were separated via reducing SDS-PAGE (except for HLA-B27 detection) 
or non-reducing SDS-PAGE (for HLA-B27 detection) and transferred to a PVDF membrane. After blocking, the blots were developed with a series of antibodies specific for mouse $\beta$-actin (Cat. No. CP01, EMD Millipore, MA, USA), caspase12 (Cat. No. 51-8104KC, BD, NJ, USA), calreticulin (Cat. No. 612137, BD, NJ, USA), BiP/Grp78 (Cat. No. 610979, BD, NJ, USA), HLA-B27 (Cat. No. MAB1285, Chemicon, CA, USA), p44/42 MAP kinase (ERK1/2) (Cat. No. 9102, Cell Signaling Technology, MA, USA), phospho-p44/42 MAP kinase (Cat. No. 9101, Cell Signaling Technology, MA, USA), p38 (Cat. No. 506123, EMD Millipore, MA, USA), or phospho-p38 MAPK (Cat. No. 9211, Cell Signaling Technology, MA, USA). Finally, the blots were hybridized with horseradish peroxidase-conjugated goat anti-rabbit IgG or anti-mouse IgG and developed using an AEC (3-amino-9-ethylcarbazole) substrate kit or visualized using a chemiluminescent substrate reagent.

\section{7. $R T-P C R$}

RNA (ribonucleic acid) was extracted and purified from treated CA46 cells using the TRIzol reagent (Cat. No. 15596-018, Ambion, CA, USA) and chloroform, according to the manufacturer's instructions. For reverse transcription, RNA samples were mixed with dNTPs, oligo(dT), and DEPC-treated water and incubated at $65 \sim 70{ }^{\circ} \mathrm{C}$ for $5 \mathrm{~min}$ and then on ice for 5 min. After incubation with buffer, RNasin ${ }^{\circledR}$ (Promega) and RTase at $70{ }^{\circ} \mathrm{C}$ for $15 \mathrm{~min}$, the samples containing cDNA (complementary deoxyribonucleic acid) were immediately chilled on ice.

To detect the alternative splicing of XBP-1, the following PCR primer sequences were used: $\beta$-actin (forward), 5'-CACTCTTCCAGCCTTCCTTCC-3'; $\beta$-actin (reverse), 5'-CGGACTCGTCATACTCCTGCTT-3'; hXBP-1 (forward), 5'-AACAGAGTAGCAGCTCAGACTGC-3'; and hXBP-1 (reverse), 5'-GGTATCTCTAAGACTAGGGGCTTGGTA-3'. The PCR temperature profile was $5 \mathrm{~min}$ at $94{ }^{\circ} \mathrm{C}$ (initial melt); 27 cycles of $45 \mathrm{~s}$ at $94{ }^{\circ} \mathrm{C}, 45 \mathrm{~s}$ at $58{ }^{\circ} \mathrm{C}$, and $80 \mathrm{~s}$ at $72{ }^{\circ} \mathrm{C}$; and $10 \mathrm{~min}$ at $72{ }^{\circ} \mathrm{C}$. The amplified DNA fragments were separated via $3 \%$ agarose electro-phoresis in TAE (Tris-acetate-EDTA) buffer at $50 \mathrm{~V}$ for $60 \mathrm{~min}$.

\section{Results and Discussion}

3.1. No Endoplasmic Reticulum Stress Induced by the Capsule Polysaccharide of Klebsiella pneumoniae in CA46 Cells

Although the molecular mimicry hypothesis regarding the induction of AS by Klebsiella was proposed nearly three decades ago, the relationship between Klebsiella infection and ERS induction remains unknown. In addition to the roles of KP in pneumonia, urethral tract infection and bacteremia, KP is a major cause of liver abscess in patients with diabetes mellitus in Taiwan, and the CPS of KP plays the predominant role in the pathogenesis of this disease $[18,20]$.

To determine whether the CPS of KP induces ERS, we extracted and purified the CPS from KP using Zwittergent. The CA46 cell line, a B cell lymphoma line obtained from a patient carrying the hla-b27 heavy chain gene, was treated with various doses of the KP CPS for $24 \mathrm{~h}$ or $48 \mathrm{~h}$. As shown in Fig. 1, the protein levels of the ERS markers BiP/Grp78 and calreticulin were not up-regulated by the KP CPS in CA46 cells. It has been suggested that a high amount of KP CPS results in an anti-phagocytic effect, which plays an important role in the pathogenesis of KP-induced liver abscess in patient with diabetes. We propose that the KP CPS may also block the interactions of bacterial components with cells in our culture system.

\subsection{Induction of ERS by Decapsulated Klebsiella pneumoniae in CA46 Cells \\ Due to the unresponsiveness of CA46 cells to the KP CPS (Fig. 1), we assessed the effect of the remaining bacterial fraction on ERS induction. Tunicamycin, an}




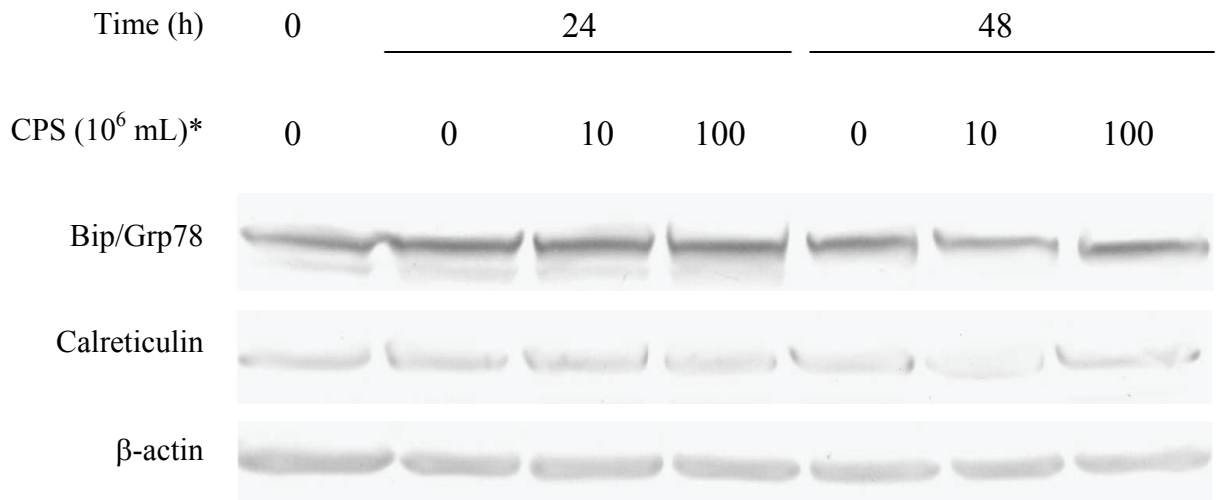

Fig. 1 The Klebsiella pneumoniae capsule polysaccharide does not induce endoplasmic reticulum stress in the HLA-B27-expressing B cell line CA46.

CA46 B lymphoma cells $\left(10^{6} / \mathrm{mL}\right)$ were treated with various doses of the capsule polysaccharide extracted from Klebsiella pneumoniae for $24 \mathrm{~h}$ or $48 \mathrm{~h}$, as described in the Materials and Methods. After incubation, the BiP/Grp78, calreticulin and $\beta$-actin levels were detected through Western blot analysis. *The indicated doses of capsule polysaccharide (CPS) represent the amount of CPS in the culture extracted from the indicated concentration of bacteria.

ERS inducer that blocks N-linked glycosylation (N-glycans), enhanced BiP/Grp78 and calreticulin protein expression in CA46 cells (Fig. 2a). Interestingly, decapsulated KP also enhanced $\mathrm{BiP} / \mathrm{Grp} 78$ and calreticulin protein expression, in a dose-dependent manner (Figs. 2a and 2b). In addition, pro-caspase 12, an ERS-activated caspase, was degraded in CA46 cells treated with a high dose of decapsulated KP (Fig. 2b).

The alternative splicing of XBP-1 mRNA has been implicated in the ERS-triggered signaling pathway, and spliced XBP-1 contributes to activation of the UPR (unfolded protein response) [21]. As shown in Fig. 3, RT-PCR analysis revealed that the level of spliced XBP-1 mRNA was increased in CA46 cells treated with decapsulated KP or tunicamycin.

To examine the other signaling pathways involved in ERS/UPR, MAPK kinase p38 activity was evaluated. The level of phosphorylated p38 (active form) was significantly increased in CA46 cells in the KP MOI 10 and 100 groups and the tunicamycin-treated group in a dose-dependent manner. In contrast, the phosphorylation of ERK kinase was decreased (Fig. 4).

\subsection{Induction of Toll-like Receptor-MyD88 Signaling} Pathway-Dependent ERS by Klebsiella pneumoniae

Although the molecular mimicry theory regarding the contribution of Klebsiella to the pathogenesis of AS was proposed several decade ago, the relationship between the non-antigenic components of Klebsiella and ERS remains unclear.

The TLR family belongs to the PRRs, a group of receptors in the immune system that respond to microbe infections [22]. E. coli LPS triggers ERS in the macrophage cell line RAW 264.7 [23], and the TLR2/4 complex and TLR7 contribute to UPR activation and sustained production of proinflammatory cytokines in macrophages [24, 25]. However, whether TLR9 activates the expression of genes downstream of ERS is unknown.

To determine the importance of the TLR-MyD88 signaling pathway in decapsulated Klebsiella pneumoniae-induced ERS, we first treated splenocytes obtained from wild-type control mice (C57BL/6 strain) or MyD88 gene knockout mice with decapsulated KP bacteria, KP LPS, or KP gDNA. Splenocytes from wild-type mice exhibited increased BiP/Grp78 and calreticulin protein expression after induction with 
decapsulated KP bacteria, KP LPS, or KP gDNA. However, the induction of both ERS markers by decapsulated KP bacteria, KP LPS, or KP gDNA was significantly suppressed in MyD88 knockout splenocytes (Fig. 5).
Because LPS triggers UPR signaling pathways and a connection between TLR9 and ERS has not been demonstrated, we sought to determine whether ERS is induced by TLR9 using TLR9 knockout mice. As shown in Fig. 6, BiP/Grp78 protein expression was

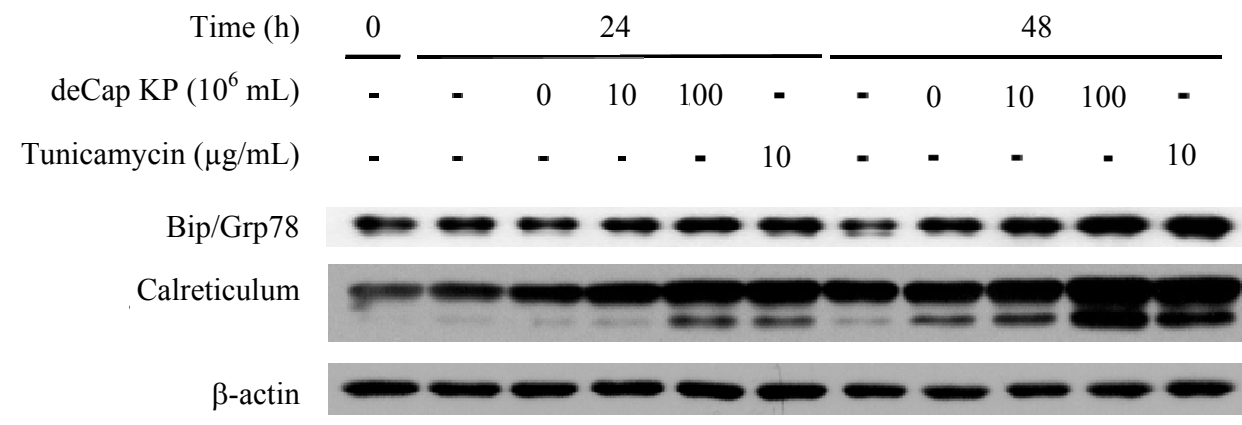

(a)

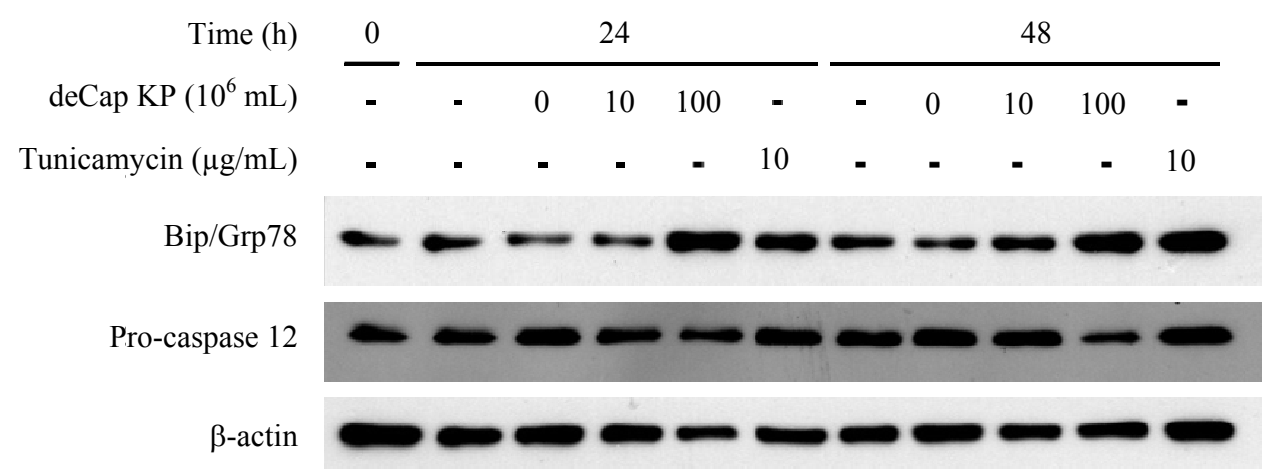

(b)

Fig. 2 Decapsulated Klebsiella pneumoniae induces ER stress and pro-caspase 12 activation in CA46 cells in a dose-dependent manner: (a) calreticulin; (b) pro-caspase12.

CA46 cells $\left(10^{6} / \mathrm{mL}\right)$ were treated with the indicated doses of decapsulated Klebsiella pneumoniae for $24 \mathrm{~h}$ or $48 \mathrm{~h}$, as described in the Materials and Methods. After incubation, the BiP/Grp78, calreticulin, pro-caspase12 and $\beta$-actin levels were detected through Western blot analysis. Tunicamycin treatment was used as the positive control for ER stress induction.

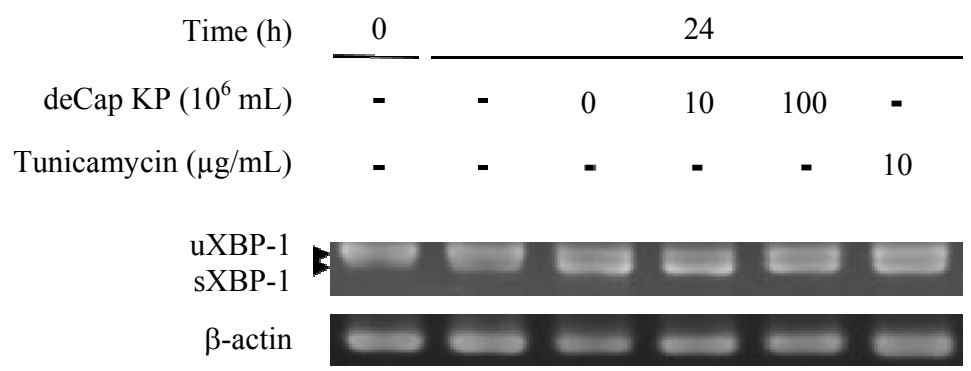

Fig. 3 Reverse transcription-PCR analysis for the identification of XBP-1 alternative splicing in decapsulated Klebsiella pneumoniae-treated CA46 cells.

CA46 cells $\left(10^{6} / \mathrm{mL}\right)$ were treated with the indicated doses of decapsulated Klebsiella pneumoniae for $24 \mathrm{~h}$, as described in the Materials and Methods. RNA obtained from untreated and 24-h-treated cells was used for RT-PCR, and the unspliced and spliced XBP-1 signals were detected via agarose gel electrophoresis. Primers specific for XBP-1 confirmed the splicing of XBP-1 RNA after treatment with decapsulated Klebsiella pneumoniae $(24 \mathrm{~h})$ or tunicamycin $(10 \mu \mathrm{g} / \mathrm{mL}, 24 \mathrm{~h})$. 


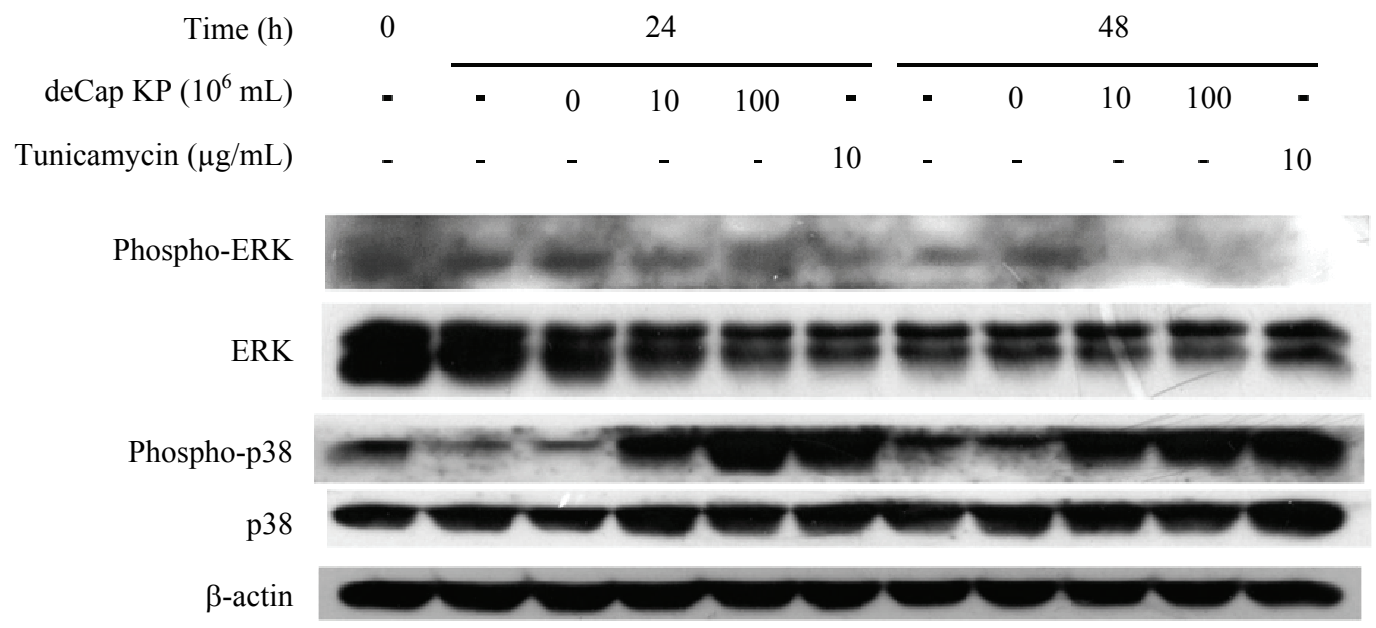

Fig. 4 Decapsulated Klebsiella pneumoniae induces p38 activation and suppresses ERK activity.

CA46 cells $\left(10^{6} / \mathrm{mL}\right)$ were treated with the indicated doses of decapsulated Klebsiella pneumoniae for $24 \mathrm{~h}$ or $48 \mathrm{~h}$. After incubation, the phospho-ERK, phospho-p38, ERK, and p38 levels were detected through Western blot analysis. Tunicamycin treatment was used as the positive control for ER stress induction.

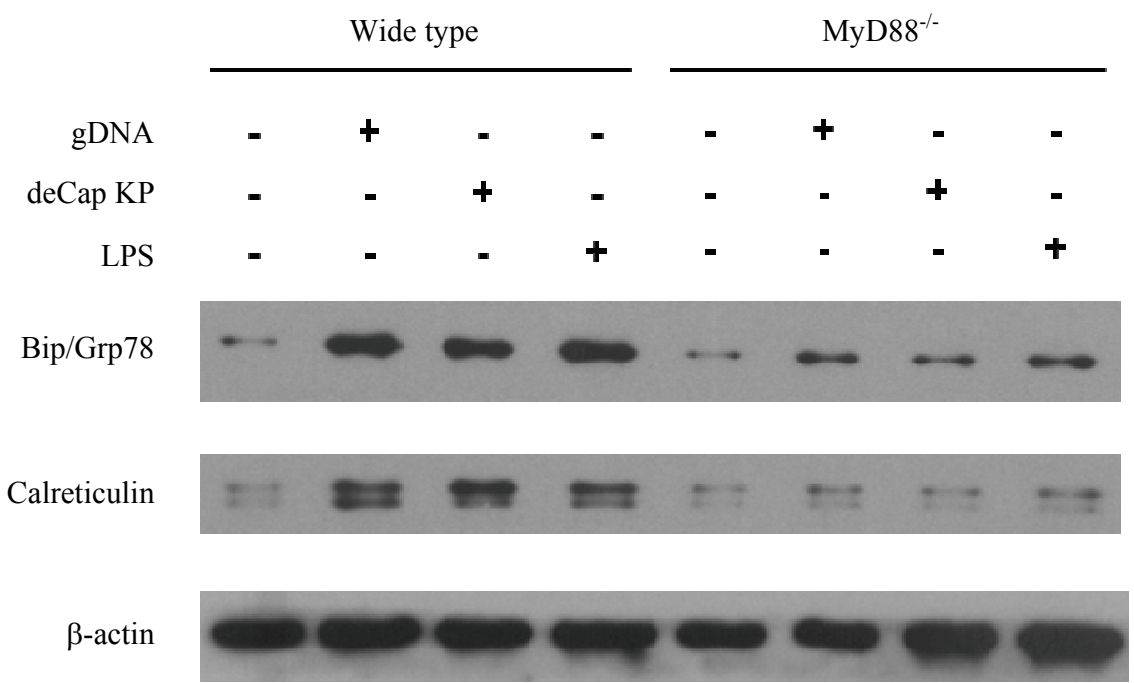

Fig. 5 MyD88 knockout decreases decapsulated Klebsiella pneumoniae-induced ER stress.

Splenocytes $\left(10^{6} / \mathrm{mL}\right)$ obtained from wild-type and MyD88 knockout mice were treated with decapsulated Klebsiella pneumoniae $\left(10^{8} / \mathrm{mL}\right)$ or with KP genomic DNA and KP lipopolysaccharide extracted from the same amount of bacteria. After a 24-h incubation, $\mathrm{BiP} / \mathrm{Grp} 78$, calreticulin and $\beta$-actin levels were detected through Western blot analysis.

induced by decapsulated KP bacteria and KP gDNA in a dose-dependent manner in splenocytes obtained from wild-type C57BL/6 mice, but this effect was not observed in splenocytes from TLR9 knockout mice.

Turner and colleagues detected enhancement of HLA-B27 expression and UPR induction in LPS-activated macrophages derived from HLA-B27/h $\beta_{2} \mathrm{~m}$ transgenic rats [26]. A similar report indicated that the induction of HLA-B27 heavy chain-homodimers was detected in HLA-B27-expressing dendritic cells after PMA/ionomycin stimulation [27]. Both studies demonstrated that activation of HLA-B $27^{+}$ antigen-presenting cells may cause ERS and B27 misfolding. In contrast, the gene expression profiles observed after LPS stimulation differed between monocyte-derived dendritic cells from HLA-B27 ${ }^{+}$ patients with AS and those from controls [28, 29]. We 

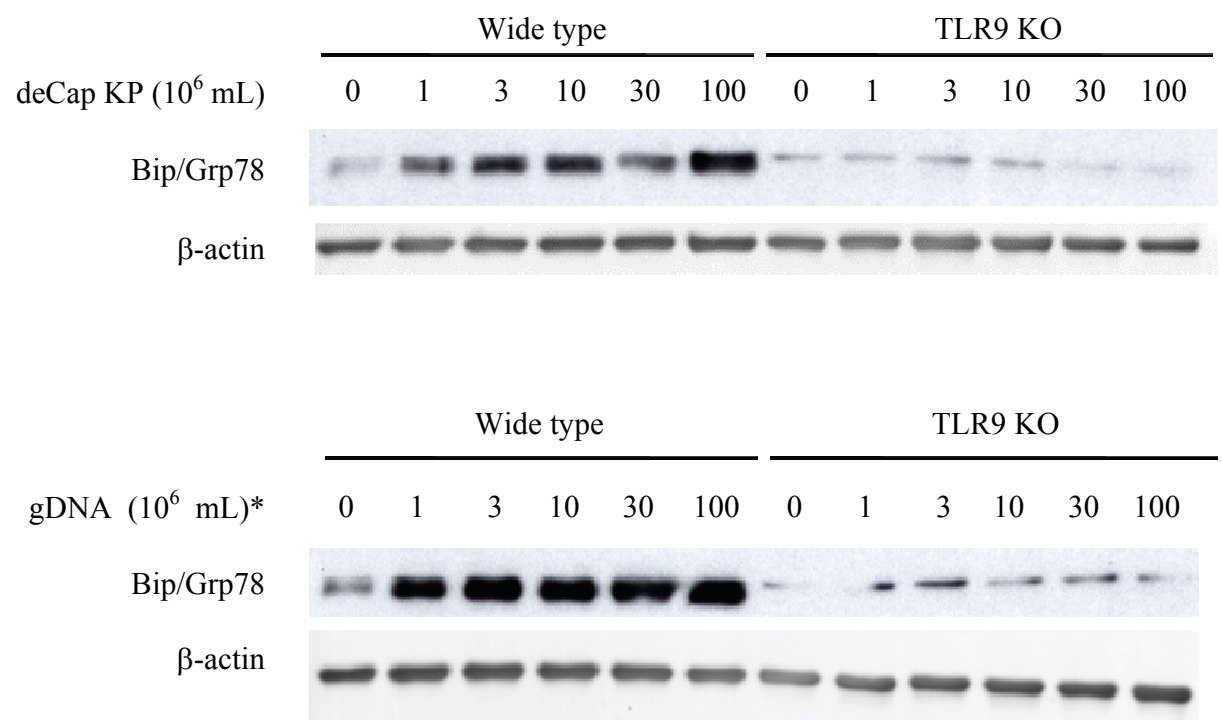

Fig. 6 TLR9 knockout decreases decapsulated Klebsiella pneumoniae-induced ER stress.

Splenocytes obtained from wild-type and TLR9 knockout mice were treated with the indicated concentrations of decapsulated Klebsiella pneumoniae or with KP genomic DNA in an amount equivalent to that extracted from the same amount of bacteria. After a 24-h incubation, BiP/Grp78 and $\beta$-actin levels were detected through Western blot analysis.

proposed that the pattern of microbiota in patients with AS might differ from that of control individuals. In fact, a germ-free environment might prevent B27-associated gut inflammation and reactive arthritis in B27 transgenic rats [30]. Based on the above-described investigations, that existence of pathogenic microbes might cause B27 heavy chain misfolding through immune cell activation. In the present study, decapsulated KP bacterial bodies or KP components (gDNA or LPS) significantly induced BiP/Grp78 expression in the splenocytes of wild-type mice, and this induction was significantly reduced in MyD88and TLR-9-knockout mice. Our results strongly suggest that activation by microbes or PAMPs might be important for the induction of the B27-related pathogenesis of spondyloarthropathy and might explain why only $5 \%$ of B27-bearing individuals have AS.

\subsection{Induction of HLA-B27 Heavy Chain Dimerization by Decapsulated Klebsiella pneumoniae}

HLA-B27 heavy chain misfolding was previously suggested to be an inducer of ERS and might play a role in the pathogenesis of AS [10]; however, the misfolding of the B27 heavy chain and the role of misfolded B27 heavy chains in the pathogenesis of AS remain unclear. Because our results demonstrated that KP triggers ERS, it is possible that B27 heavy chain misfolding also requires an initiating event. Therefore, we determined whether B27 heavy chain misfolding is triggered by KP. We treated CA46 cells with various doses of decapsulated KP for $24 \mathrm{~h}$, as described previously, and then detected the misfolded HLA-B27 heavy chain complexes by SDS-PAGE under non-reducing conditions (without DTT and $\beta$-mercaptoethanol) and Western blotting. As shown in Fig. 7, a high dose of decapsulated KP bacteria significantly induced the misfolded form of the B27 heavy chain. Interestingly, although tunicamycin induces ERS (Figs. 2-4), we did not observe misfolded HLA-B27 heavy chain complexes in the presence of tunicamycin (Fig. 7). Furthermore, we also found that decapsulated KP induced ERS in the RAW 264.7 cell line, which does not express HLA-B27 (our unpublished data). In conclusion, KP-triggered ERS-related signaling pathways and gene expression are not necessarily induced by HLA-B27 misfolding. Conversely, KP-triggered ERS appears to promote HLA-B27 misfolding. 


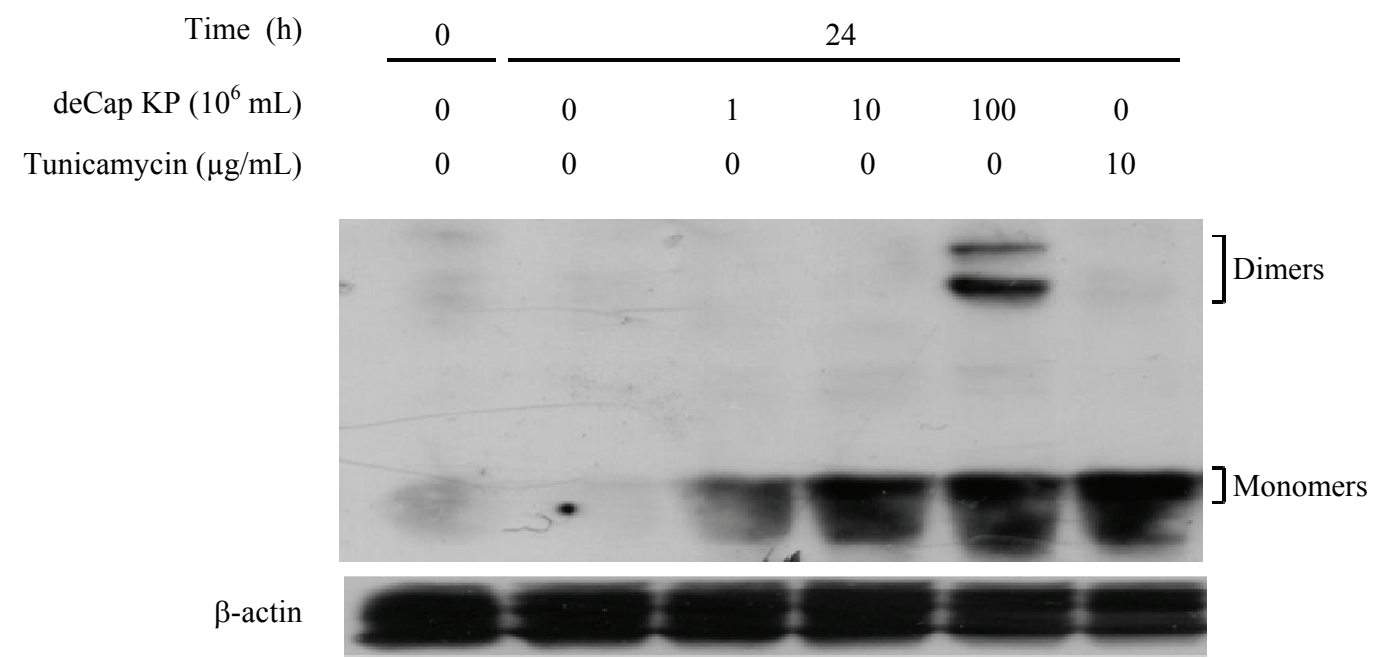

Fig. 7 Induction of HLA-B27 homodimer formation in CA46 B lymphocytes treated with decapsulated Klebsiella pneumoniae.

CA46 cells $\left(10^{6} / \mathrm{mL}\right)$ were treated with the indicated doses of decapsulated Klebsiella pneumoniae for $24 \mathrm{~h}$. After incubation, the levels of monomeric and dimeric HLA-B27 heavy chains were detected via non-reducing gel electrophoresis and Western blot analysis. Tunicamycin treatment was used as the positive control for ER stress induction.

Klebsiella infection has been proposed as a pathological environmental risk factor that contributes to the pathogenesis of AS [31], but the connection between Klebsiella and recent AS pathogenic models including ERS and HLA-B27 heavy chain misfolding remains unclear.

Our results indicated that KP CPS is being unable to induce ERS in B cell results from the blocking of $B$ cell activation caused by other KP components. Although the use of de-capsulation enabled elucidation of the immunostimulatory effect of KP in our experiments, the mechanism through which KP component(s) increases immune cell activation in vivo remains unclear.

Rashid et al. reported that the anti-KP antibody levels were significantly elevated in patients with AS in 16 different countries and that the fecal isolation rates of Klebsiella were increased in AS patients [32]. Measurements of anti-KP antibodies in the serum or feces might be an indicator of the active state of AS, but whether anti-KP antibodies enhance the engulfment of KP and are associated with AS severity remains to be elucidated.

We initially used the B lymphoma cell line CA46 to examine the effects of KP or its components on the induction of ERS based on the data sheet provided by the ATCC, which indicated this cell line expresses HLA-B27. However, to demonstrate the role of the TLR/MyD88 signaling pathway in ERS induction, immune cells (splenocytes) collected from gene-knockout mice with the hla-b2 $7^{-/}$phenotype were used. In addition, an identical phenomenon of ERS induction was observed in decapsulated KP-treated macrophage-like U937 cells (unpublished data), but the U937 cells do not express HLA-B27. These results again demonstrated that HLA-B27 misfolding is not necessary for ERS induction. As previously reported, ERS is enhanced in cytokine-differentiated marrow-derived dendritic cells and macrophages from HLA-B $27^{+}$patients or B27 transgenic rats after LPS stimulation $[26,27]$. Our results demonstrated that KP LPS and gDNA also induce ERS in splenocytes not expressing HLA-B27. We concluded that ERS is induced by PAMPs in all types of professional antigen-presenting cells, including $\mathrm{B}$ cells and this phenomenon might affect antigen presentation.

B lymphocyte activation has been demonstrated in AS patients. The levels of antibodies against the KP 
capsule and LPS are increased in patients with AS. A recent study indicated that the subset profiles of $B$ lymphocytes of patients with AS is different from that of healthy controls, and the magnitude of the difference is correlated with BASDI (Bath Ankylosing Spondylitis Disease Activity Index) [33-35]. Based on the above findings and our observation of the induction of ERS in CA46 cells by KP LPS or gDNA, we suggest that humoral immune responses against KP are activated in patients with AS. However, whether antibodies against KP LPS might block non-antigen-dependent B cell activation through the TLR4 signal pathway or contrarily facilitate immune complex formation and thereby helping engulfment remains to be elucidated. Furthermore, whether phagocytosis results in the release of gDNA from the engulfed and destroyed KP cells and then the induction TLR9/MyD88 signaling and ERS also needs to be clarified.

An increasing number of studies are providing insights into the origin of inflammation in AS patients. Pöllänen et al. [36] proposed that PAMPs released from bacteria localize to biofilms in urogenital organs and that DAMPs from damaged cells are transported through lymphatic vessels to the spine. They held the view that both PAMP and DAMP molecules might stimulate TLRs in mesenchymal stem cells to undergo ectopic enchondral bone formation and lead to the development of bamboo spine [36]. Our results indirectly support the hypothesis that TLR activation is essential in the pathogenesis of AS. A recent review article indicated that plasma cells not only produce antibodies but also release cytokines, including IL-35, IL-10, TNF- $\alpha$, GM-CSF, and IL-17. IgA-producing plasma cells also release iNOS, and the induction of UPR can prolong the lifespan of plasma cells [37]. In addition, CXCR4/CXCL12 chemotaxis guides the migration of activated $\mathrm{B}$ cell into bone marrow. Interestingly, XBP-1 deficiency might result in a loss of bone marrow colonization of $\mathrm{B}$ cells after activation through an ineffective response to to CXCL12 [38, 39]. Whether the stimulation of UPR is activated $\mathrm{B}$ lymphocytes by bacterial components results in bone marrow migration and spinal inflammation in patients with AS is an interested topic.

Importantly, immune cell stimulation due to TLR signaling is a not a KP-specific phenomenon. Why is Klebsiella spp. the major candidate microbe for the pathogenesis of AS? The oldest theory is based on the molecular mimicry of bacterial antigens, which is supported by data from clinical investigations [13, 40]. Our findings demonstrate that both TLR9 and MyD88 are important in ERS-related signaling, gene induction and HLA-B27 misfolding. However, additional research is necessary to determine whether ERS through TLR signaling activation aids KP antigen presentation by misfolded B27 heavy chains, and subsequently activates autoreactive T cells or NK cells.

\section{Conclusions}

Our study demonstrated that both ERS induction and HLA-B27 misfolding in B cells can be caused by the triggering of decapsulated KP. This study provides the first connection of the role of KP with a recent pathogenic hypothesis in AS. In addition, we found that activation of the TLR9/MyD88 signaling pathway is involved in KP-mediated ERS induction. These effects establish a potential role for autoreactive $\mathrm{T}$ cell or NK cell activations via structural changes in the HLA-B27 heavy chain or the expression of a different antigen profile on antigen-presenting cells. This finding also breaks down the long-existing stereotypical conceptions that suggest that TLR pathway is activated mainly to trigger the induction of an innate immune response and that the activation of B lymphocytes mainly leads to the generation of antibody-producing cells.

Recent medicines for AS treatment include NSAIDs (non-steroid anti-inflammatory drugs), biphosphates, disease-modifying antirheumatic drugs (DMARDs), thalidomide, and anti-TNF (tumor necrosis factor) antibodies. Most of these drugs reduce the inflammatory state or modulate autoimmune responses, 
but none interrupts the initial immune activation. Based on our findings, we conclude that suppressing the immune response using a TLR antagonist or replacing pathogenic KP by probiotics might be of therapeutic and/or prophylactic potential in $\mathrm{AS}$, and we strongly advocate these treatment approaches.

\section{Acknowledgments}

We thank Dr. Kuei-Tai Lai for providing the TLR9 and MyD88-knockout mice.

The present study was supported by Grant CSMU-TSMH-098-003 from Chung Shan Medical University and Antai Tian-Sheng Memorial Hospital, Taiwan.

\section{Disclosure statement}

All authors have declared no conflicts of interest.

\section{References}

[1] Wang, L., Wang, F. S., and Gershwin, M. E. 2015. "Human Autoimmune Diseases: A Comprehensive Update." J. Intern. Med. 278: 369-95.

[2] Pásztói, M., Misják, P., György, B., Aradi, B., Szabó, T. G., Szántó, B., et al. 2011. "Infection and Autoimmunity: Lessons of Animal Models.” Eur. J. Microbiol. Immunol. (BP) 1: 198-207.

[3] Wu, H. J., Ivanov, I. I., Darce, J., Hattori, K., Shima, T., Umesaki, Y., et al. 2010. "Gut-Residing Segmented Filamentous Bacteria Drive Autoimmune Arthritis via T Helper 17 Cells." Immunity 32: 815-27.

[4] Chen, J. Q., Szodoray, P., and Zeher, M. 2016. "Toll-Like Receptor Pathways in Autoimmune Diseases." Clin. Rev. Allergy Immunol. 50: 1-17.

[5] Broggi, A., and Granucci, F. 2015. "Microbe- and Danger-Induced Inflammation." Mol. Immunol. 63: 127-33.

[6] Robinson, P. C., and Benham, H. 2015. "Advances in Classification, Basic Mechanisms and Clinical Science in Ankylosing Spondylitis and Axial Spondyloarthritis." Intern. Med. J. 45: 127-33.

[7] Tan, S., Wang, R., and Ward, M. M. 2015. "Syndesmophyte Growth in Ankylosing Spondylitis." Curr. Opin. Rheumatol. 27: 326-32.

[8] Brown, M. A., Kennedy, L. G., MacGregor, A. J., Darke, C., Duncan, E., Shatford, J. L., et al. 1997. "Susceptibility to Ankylosing Spondylitis in Twins: The Role of Genes, HLA, and the Environment." Arthritis Rheum 40: 1823-8.
[9] Brown, M. A., Laval, S. H., Brophy, S., and Calin, A. 2000. "Recurrence Risk Modelling of the Genetic Susceptibility to Ankylosing Spondylitis." Ann. Rheum. Dis. 59: 883-6.

[10] Sorrentino, R., Böckmann, R. A., and Fiorillo, M. T. 2014. "HLA-B27 and Antigen Presentation: at the Crossroads between Immune Defense and Autoimmunity." Mol. Immunol. 57: 22-7.

[11] Evans, D. M., Spencer, C. C., Pointon, J. J., Su, Z., Harvey, D., Kochan, G., et al. 2011. "Interaction between ERAP1 and HLA-B27 in Ankylosing Spondylitis Implicates Peptide Handling in the Mechanism for HLA-B27 in Disease Susceptibility." Nat. Genet. 43: 761-7.

[12] Uddin, M., Codner, D., Hasan, S. M., Scherer, S. W., O'Rielly, D. D., and Rahman, P. 2015. "Integrated Genomics Identifies Convergence of Ankylosing Spondylitis with Global Immune Mediated Disease Pathways." Sci. Rep. 5: 10314.

[13] Ebringer, R. W., Cawdell, D. R., Cowling, P., and Ebringer, A. 1978. "Sequential Studies in Ankylosing Spondylitis. Association of Klebsiella Pneumoniae with Active Disease." Ann. Rheum Dis. 37: 146-51.

[14] Fielder M, Pirt SJ, Tarpey I, Wilson C, Cunningham P, Ettelaie C, et al. 1995. "Molecular Mimicry and Ankylosing Spondylitis: Possible role of a Novel Sequence in Pullulanase of Klebsiella pneumoniae." FEBS Lett. 369: 243-8.

[15] Schittenhelm, R. B., Sian, T. C., Wilmann, P. G., Dudek, N. L., and Purcell, A. W. 2015. "Revisiting the Arthritogenic Peptide Theory: Quantitative not Qualitative Changes in the Peptiderepertoire of HLA-B27 Allotypes." Arthritis Rheumatol. 67: 702-13.

[16] Smith, J. A. 2015. "The Role of the Unfolded Protein Response in Axial Spondyloarthritis." Clin. Rheumatol. 35: 1425-31.

[17] Podschun, R., and Ullmann, U. 1998. “Klebsiella spp. as Nosocomial Pathogens: Epidemiology, Taxonomy, Typing Methods, and Pathogenicity Factors." Clin. Microbiol. Rev. 11: 589-603.

[18] Lin, J. C., Chang, F. Y., Fung, C. P., Xu, J. Z., Cheng, H. P., Wang, J. J., et al. 2004. "High Prevalence of Phagocytic-Resistant Capsular Serotypes of Klebsiella pneumoniae in Liver Abscess." Microbes Infect. 6: 1191-8.

[19] Pöllänen, R., Sillat, T., Pajarinen, J., Levón, J., Kaivosoja, E., and Konttinen, Y. T. 2009. "Microbial Antigens Mediate HLA-B27 Diseases via TLRs.” J. Autoimmun. 32: 172-7.

[20] Tsai, F. C., Huang, Y. T., Chang, L. Y., and Wang, J. T. 2008. "Pyogenic Liver Abscess as Endemic Disease, Taiwan." Emerg. Infect. Dis. 14: 1592-600.

[21] Yoshida, H., Matsui, T., Yamamoto, A., Okada, T., and 
Mori, K. 2001. "XBP1 mRNA is Induced by ATF6 and Spliced by IRE1 in Response to ER Stress to Produce a Highly Active Transcription Factor.” Cell 107: 881-91.

[22] Takeuchi, O., and Akira, S. 2010. "Pattern Recognition Receptors and Inflammation." Cell 140: 805-20.

[23] Gotoh, T., Oyadomari, S., Mori, K., and Mori, M. 2002. "Nitric Oxide-Induced Apoptosis in RAW 264.7 Macrophages is Mediated by Endoplasmic Reticulum Stress Pathway Involving ATF6 and CHOP.” J. Biol. Chem. 277: 12343-50.

[24] Martinon, F., Chen, X., Lee, A. H., and Glimcher, L. H. 2010. "TLR Activation of the Transcription Factor XBP1 Regulates Innate Immune Responses in Macrophages.” Nat. Immunol. 11: 411-8.

[25] Yu, X., Wang, Y., Zhao, W., Zhou, H., Yang, W., and Guan, X. 2014. "Toll-Like Receptor 7 Promotes the Apoptosis of THP-1-Derived Macrophages through the CHOP-Dependent Pathway." Int. J. Mol. Med. 34: 886-93.

[26] Turner, M. J., Sowders, D. P., DeLay, M. L., Mohapatra, R., Bai, S., Smith, J. A., et al. 2005. "HLA-B27 Misfolding in Transgenic Rats is Associated with Activation of the Unfolded Protein Response." J. Immunol. 175: 2438-48.

[27] Santos, S. G., Lynch, S., Campbell, E. C., Antoniou, A. N., and Powis, S. J. 2008. "Induction of HLA-B27 Heavy Chain Homodimer Formation after Activation in Dendritic Cells." Arthritis Res. Ther. 10: R100.

[28] Slobodin, G., Kessel, A., Kofman, N., Toubi, E., Rosner, I., and Odeh, M. 2012. "Phenotype of Resting and Activated Monocyte-Derived Dendritic Cells Grown from Peripheral Blood of Patients with Ankylosing Spondylitis." Inflammation 35: 772-5.

[29] Talpin, A., Costantino, F., Bonilla, N., Leboime, A., Letourneur, F., Jacques, S., et al. 2014. "Monocyte-Derived Dendritic Cells from HLA-B27+ Axial Spondyloarthritis (SpA) Patients Display Altered Functional Capacity and Deregulated Gene Expression." Arthritis Res. Ther. 16: 417.

[30] Taurog, J. D., Richardson, J. A., Croft, J. T., Simmons, W. A., Zhou, M., Fernández-Sueiro, J. L., et al. 1994. "The
Germfree State Prevents Development of Gut and Joint Inflammatory Disease in HLA-B27 Transgenic Rats." $J$. Exp. Med. 180: 2359-64.

[31] Ebringer, R., Cooke, D., Cawdell, D. R., Cowling, P., and Ebringer, A. 1977. "Ankylosing Spondylitis: Klebsiella and HL-A B27." Rheumatol. Rehabil. 16: 190-6.

[32] Rashid, T., Ebringer, A., and Wilson, C. 2016. "The Link between Klebsiella and Ankylosing Spondylitis in Worldwide Geographical Locations." Curr. Rheumatol. Rev. (Epub ahead of print)

[33] Sahly, H., Podschun, R., Kekow, J., Nölle, B., Gross, W. L., and Ullmann, U. 1998. "Humoral Immune Response to Klebsiella Capsular Polysaccharides in HLA-B27-Positive Patients with Acute Anterior Uveitis and Ankylosing Spondylitis." Autoimmune 28: 209-15.

[34] Ahmadi, K., Wilson, C., Tiwana, H., Binder, A., and Ebringer, A. 1998. “Antibodies to Klebsiella pneumoniae Lipopolysaccharide in Patients with Ankylosing Spondylitis.” Br. J. Rheumatol. 37: 1330-3.

[35] Niu, X. Y., Zhang, H. Y., Liu, Y. J., Zhao, D., Shan, Y. X., and Jiang, Y. F. 2013. "Peripheral B-Cell Activation and Exhaustion Markers in Patients with Ankylosing Spondylitis." Life Sci. 93: 687-92.

[36] Pöllänen, R., Sillat, T., Pajarinen, J., Levón, J., Kaivosoja, E., and Konttinen, Y. T. 2009. "Microbial Antigens Mediate HLA-B27 Diseases via TLRs.” J. Autoimmun. 32: 172-7.

[37] Gommerman, J. L., Rojas, O. L., and Fritz, J. H. 2014. "Re-thinking the Functions of $\operatorname{IgA}(+)$ Plasma Cells." Gut Microbes 5: 652-62.

[38] Hargreaves, D. C., Hyman, P. L., Lu, T. T., Ngo, V. N., Bidgol, A., Suzuki, G., et al. 2001. "A Coordinated Change in Chemokine Responsiveness Guides Plasma Cell Movements." J. Exp. Med. 194: 45-56.

[39] Hu, C. C., Dougan, S. K., McGehee, A. M., Love, J. C., and Ploegh, H. L. 2009. "XBP-1 Regulates Signal Transduction, Transcription Factors and Bone Marrow Colonization in B Cells.” EMBO J. 28: 1624-36.

[40] Rashid, T., and Ebringer, A. 2007. "Ankylosing Spondylitis is Linked to Klebsiella—The Evidence." Clin. Rheumatol. 26: 858-64. 